\title{
Objective perimetry using a four-channel multifocal VEP system: correlation with conventional perimetry and thickness of the retinal nerve fibre layer
}

\author{
Folkert K Horn, Christoph Kaltwasser, Anselm G Jünemann, Jan Kremers, \\ Ralf $P$ Tornow
}

Department of Ophthalmology, University of Erlangen-Nürnberg, Erlangen, Germany

\section{Correspondence to}

Folkert Horn, Department of Ophthalmology and University Eye Hospital,

Friedrich-Alexander University of Erlangen-Nürnberg,

Schwabachanlage 6, 91054

Erlangen, Germany;

folkert.horn@uk-erlangen.de

Accepted 2 November 2011

Published Online First

24 November 2011

\section{ABSTRACT}

Purpose There is evidence that multifocal visual evoked potentials (VEPs) can be used as an objective tool to detect visual field loss. The aim of this study was to correlate multifocal VEP amplitudes with standard perimetry data and retinal nerve fibre layer (RNFL) thickness.

Method Multifocal VEP recordings were performed with a four-channel electrode array using 58 stimulus fields (pattern reversal dartboard). For each field, the recording from the channel with maximal signal-to-noise ratio (SNR) was retained, resulting in an SNR optimised virtual recording. Correlation with RNFL thickness, measured with spectral domain optical coherence tomography and with standard perimetry, was performed for nerve fibre bundle related areas.

Results The mean amplitudes in nerve fibre related areas were smaller in glaucoma patients than in normal subjects. The differences between both groups were most significant in mid-peripheral areas. Amplitudes in these areas were significantly correlated with corresponding RNFL thickness (Spearman $\mathrm{R}=0.76$ ) and with standard perimetry $(R=0.71)$.

Conclusion The multifocal VEP amplitude was correlated with perimetric visual field data and the RNFL thickness of the corresponding regions. This method of SNR optimisation is useful for extracting data from recordings and may be appropriate for objective assessment of visual function at different locations. Trial registration number This study has been registered at http://www.clinicaltrials.gov (NCT00494923).

\section{INTRODUCTION}

Visual evoked potential (VEP) measurement allows objective assessment of visual function in ocular and systemic disease. ${ }^{1}$ VEP recording during multifocal stimulation has been suggested to be an objective perimetric method. ${ }^{2}$ The technique of pattern reversal multifocal VEP (mFVEP) offers the possibility for such a test. mfVEPs can be recorded from the skull by placing electrodes at different positions. ${ }^{3}$ It was shown that this method can be used to asses the visual field in the central $20^{\circ}$ to $30^{\circ}$ in patients with optic nerve disorders, glaucoma and hemianopsia, and that it might be sensitive for detecting visual field loss. ${ }^{4-9}$ A topographical association between mfVEP and structural damage has been established for patients with opticus neuritis, glaucoma and multiple sclerosis. ${ }^{5} 6$ 10-12 However, inter-individual differences and recording variability sometimes make use of the mfVEP technique difficult. ${ }^{13}$ However, the use of multielectrode configurations to record the mfVEP signals improves the sensitivity of the method. ${ }^{3} \mathrm{We}$ present an analysis technique which optimises the signal-to-noise ratio (SNR) from a four-channel recording system to extract reliable data.

The purpose of this study was to determine whether the results of visual field tests obtained with a four-channel mfVEP system are comparable to those obtained by standard automated perimetry. These comparisons were performed in glaucoma patients with perimetric and optic nerve defects. Subsequently, we explored how the VEP amplitudes of nerve fibre related areas differ between normal subjects and glaucoma patients, and how they are related to structural defects revealed by spectral optical coherence tomography (SOCT).

\section{METHODS}

\section{Experimental design and stimulus}

A standard pattern reversal dartboard stimulus was presented on a 19-inch TFT flat-screen (RETIsystem; Roland Consult, Brandenburg, Germany). Visual stimuli were presented monocularly in eight cycles of $140 \mathrm{~s}$ each. The optimally refracted patients were instructed to fixate a cross (size: $1^{\circ}$ ). Correct visual fixation was monitored with a video camera system. VEPs were recorded with four different electrodes placed on the subject's skull using a cross-shaped electrode fixation device (electrode distance from inion: above, $3.5 \mathrm{~cm}$; below, $3 \mathrm{~cm}$; horizontal left and right, $4 \mathrm{~cm}$ ). ${ }^{9}$ The joint mounting of the electrodes ensured an equal distribution of their pressure and an improved electrode-skin contact. Thus, four differential recordings from electrode pairs with different orientations were derived. Biosignals were digitised with $1 \mathrm{kHz}$ sampling frequency and band-passed filtered with 1 and $100 \mathrm{~Hz}$ cut-off frequencies. The signal data were processed with the system's online analysis software and were subsequently further analysed off-line with MATLAB-based signal processing software written in-house. As a criterion for signal quality, SNRs were calculated for all 58 stimulated fields and for each of the four channels. The SNR was computed by dividing the signal in a time window between 50 and $150 \mathrm{~ms}$ (in which the VEP signal is expected to appear) by the signal between 230 and $330 \mathrm{~ms}$ (in which no response is elicited by the stimulus). The signal was defined as the difference between the maximal and 
minimal value measured in the above time windows. In the patient cohort the mean SNR was $2.2 \pm 0.5$ (range: 1.4-3.7), while in healthy volunteers the mean SNR was $2.5 \pm 0.8$ (1.5-4.6). The recording with maximal SNR was used in statistical analysis. For statistical analysis in nerve fibre related areas, the mean amplitudes of the single fields in these areas were calculated (figure 1).

\section{Procedures}

Informed consent, including agreement for data collection, was obtained from participants, all of whom were members of the Erlangen Glaucoma Registry. The study followed the tenets of the Declaration of Helsinki for research involving human subjects.

All participants were thoroughly examined by slit-lamp inspection, tonometry, funduscopy, gonioscopy and papillometry. Visual fields were examined using standard and FDT perimeters (screening program: n-20-5; Zeiss Humphrey Systems, Dublin, California, USA). FDT perimeter results were scored $^{14}$ from 0 (all presentations seen at first appearance) to 68 , with all missed presentations taken account of in the test protocol. Papillometric evaluations of patients were based on $15^{\circ}$ colour optic disc photographs. Criteria for glaucoma diagnosis were an open anterior chamber angle and a glaucomatous appearance of the optic nerve head including an unusually small neuroretinal rim area in relation to the optic disc size, and a larger vertical than horizontal cup-to-disc ratio. ${ }^{15}$ All individuals had clear optic media, visual acuity of $6 / 20$ or better, and intraocular pressure (IOP) less than $23 \mathrm{~mm} \mathrm{Hg}$. Exclusion criteria were all eye diseases other than glaucoma, diabetes mellitus, and a myopic refractive error exceeding -6 diopters. Age and refractive error did not differ significantly between the normal and glaucoma groups.
The retinal nerve fibre layer (RNFL) thickness of all subjects was measured by SOCT (Spectralis, Heidelberg Engineering, Germany). Averaged B-scans were analysed automatically to determine the RNFL thickness on a circle of $3.4 \mathrm{~mm}$ diameter around the optic disc. The present statistical analysis is based on 32 equal-sized sectors (11.25 each) that take account of the dependency of the RNFL thickness on the age of the subjects. ${ }^{16}$

To assess the relationship between VEP amplitudes, visual field defects and RNFL thickness, the grid of perimetric test points was scaled to match the grid of the VEP stimuli. Eight visual field areas were defined (figure 1) and were used for the comparison of VEP amplitudes between normal subjects and glaucoma patients. To correlate visual field areas and RNFL thickness data we used a similar map as previously. ${ }^{17}$ Additionally, the present mapping takes into account that the angle between the fovea and the centre of the optic disc versus the horizon was $5.9 \pm 3.7^{\circ} .{ }^{18}$ The nasal half of the RNFL thickness measurements was excluded from correlation analyses because it largely corresponds to retinal regions outside the perimetric field. The following abbreviations and nomenclature are used for visual field areas (the corresponding nerve head sectors are given in brackets, with $0^{\circ}$ corresponding to clock hour 9 in the right eye): ci, central-inferior $\left(349^{\circ}-11^{\circ}\right)$; ni, nasal-inferior $\left(11^{\circ}-45^{\circ}\right.$ ); inf, inferior $\left(45^{\circ}-90^{\circ}\right)$; sup, superior $\left(270^{\circ}-304^{\circ}\right)$; ns, nasalsuperior $\left(304^{\circ}-326^{\circ}\right)$; cs, central-superior $\left(326^{\circ}-349^{\circ}\right)$; os and oi, outer superior and inferior visual field areas (no correspondence on the optic nerve head and excluded from comparisons). The maps of the remaining six visual field areas with corresponding sectors at the optic nerve head are shade coded in figure 1.

\section{Perimetry}

All patients underwent visual field tests with standard whiteon-white perimetry (Octopus 500; Interzeag, Schlieren,
Figure 1 Relationship between the visual evoked potential (VEP) and perimetric test grids and nerve fibre related areas at the optic nerve head for a right eye. The sketch displays the multifocal VEP (mfVEP) dartboard stimulus with two nasal wings, the 46 test positions of standard perimetry (Octopus) and a graphical representation of the optic disc. Note the inverted orientation of the optic disc segmentation compared to fundus imaging in this visual field based view. The corresponding visual field areas and the optic nerve head positions, used for correlation analyses, are shade coded. The outer visual field areas (os and oi) and the nasal half of the RNFL measurements were excluded from correlation analyses. ci, central-inferior; cs, central-superior; inf, inferior; ni, nasal-inferior; ns, nasal-superior; oi, outer inferior (black and white); os, outer superior (black and white); sup, superior.

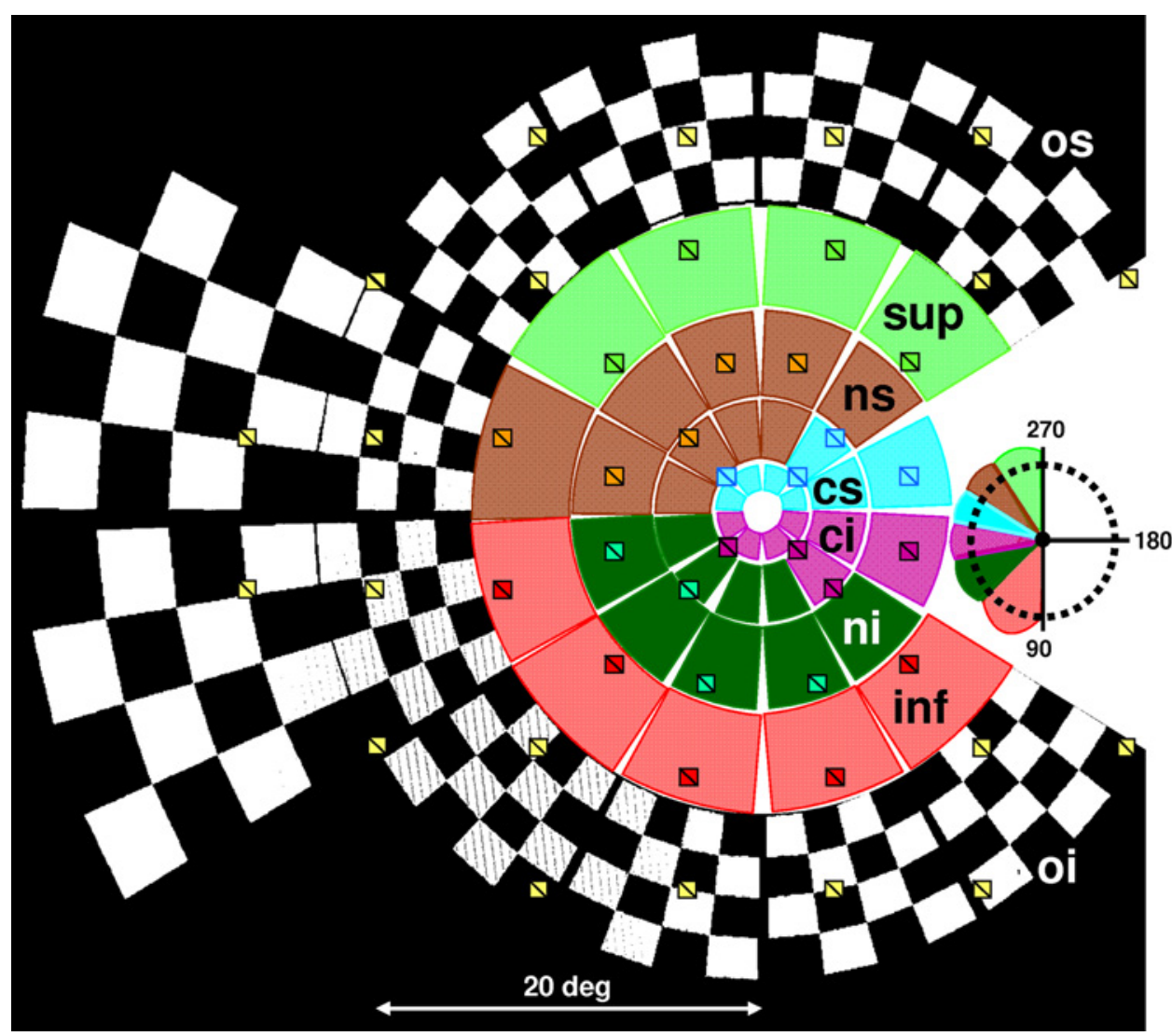


Switzerland). All patients were subject to sensory tests and were measured at least once with this perimeter. Tests with more than $12 \%$ false positive or false negative responses were excluded. The present measurement algorithm (program G1, three phases) includes 59 test positions arranged according to the path of the nerve fibres. In this study, only test locations $(n=46)$ falling within an area covered by the dartboard stimulus for VEP measurements were included in further analyses. To calculate the mean perimetric loss in nerve fibre bundle related visual field areas (figure 1), the anti-log values at all single test positions were calculated and subsequently averaged. ${ }^{19}$ For presentation and statistical analysis these averaged values were converted back to the $\mathrm{dB}$ scale.

\section{Glaucoma patients}

All patients of this group displayed glaucomatous optic disc damage as mentioned above and visual field losses. The cohort of glaucoma patients (11 female, 19 male, mean age $56.9 \pm 13.1$ years) included 13 patients with primary open-angle glaucoma characterised by elevated IOP measurements above $21 \mathrm{~mm} \mathrm{Hg}$, seven patients with secondary open-angle glaucoma with elevated IOP due to pigmentary glaucoma or pseudoexfoliation, and 10 patients with normal-tension glaucoma. If both eyes met the inclusion criteria, the eye with higher corrected loss variance (CLV) was selected for mfVEP. The mean value ( $\pm S D$ ) of perimetric defects (Octopus) in these glaucoma eyes was $6.7 \pm 4.2 \mathrm{~dB}$ and CLV was $56.2 \pm 30.7 \mathrm{~dB}^{2}$. The FDT screening score $^{14}$ was between 6 and 68 .

\section{Control group}

The study included 24 randomly selected eyes of 24 healthy subjects (six female, 18 male, mean age $52.8 \pm 13.6$ years). Slitlamp inspection, white-on-white perimetry, tonometry and funduscopy were normal without medication. The FDT screening score ${ }^{14}$ was less than or equal to 1 . Optic disc photographs were classified as normal by at least two experienced ophthalmologists.

\section{Statistical methods}

The results in control subjects and glaucoma patients are displayed in the figures as means and 95\% CIs. Results were analysed by SPSS V.18. Comparisons between groups were performed using unpaired Mann-Whitney tests. The level of significance was set at $\alpha=0.05$ for all statistical tests. Bonferroni correction for multiple testing was performed by multiplying the observed $p$ value with the number of comparisons within each analysis. Correlations between local VEP amplitudes and perimetric defects were computed for nerve fibre related visual field areas (Spearman rank correlation).

To demonstrate the relationship between sector VEP amplitudes and corresponding perimetric losses, a theoretical curve according to a linear model given by Hood et a ${ }^{20}$ was calculated and is displayed in figure 4. This model assumes that the measured voltage $U_{M}$ consists of two components: $U_{\mathrm{M}}=U_{\mathrm{A}}+U_{\mathrm{R}}$. One component $U_{\mathrm{A}}$ is the voltage generated by the stimulus, while the other component $U_{R}$ is the residual base amplitude, the noise level. As the perimetric field sensitivity decreases, the voltage $U_{\mathrm{A}}$ decreases also, while the base level $U_{\mathrm{R}}$ does not change. In this model, the voltage $U_{\mathrm{A}}$ is linearly related to the sensitivity change $S: U_{\mathrm{A}}=U_{\mathrm{A} 0} \times S$ with $U_{\mathrm{A} 0}$ the normal voltage and $S=10^{-D / 10}$, thereby converting the measured defect value $D$ (in $\mathrm{dB}$ ) to the relative sensitivity $S$ (in linear units). A normal subject is characterised by $D=0, S=1$ and $U_{\mathrm{A}}=U_{\mathrm{A} 0}$. The resulting expression for the relationship between the measured amplitude $U_{M}$ and the relative sensitivity $S$ is $U_{M}=U_{A 0} \times S+U_{R}$ and the relationship between the measured amplitude $U_{M}$ and $D$
Figure 2 Results in one glaucoma eye of a 31-year-old male patient with juvenile primary open angle glaucoma. (A) The white-on-white perimetry indicates visual field defects in the upper nasal quadrant. (B) Multifocal VEP (mfVEP) responses showing loss of signal amplitudes in the outer fields of the same quadrant. (C) Retinal nerve fibre layer profile of the same eye with the Spectralis spectral optical coherence tomography and the normative range of retinal nerve fibre layer (RNFL) thickness. An excessive reduction in RNFL thickness is found in an optic disc sector at about $270^{\circ}$ corresponding to the superior visual field. ci, central-inferior; cs, centralsuperior; inf, inferior; ni, nasal-inferior; ns, nasal-superior; oi, outer inferior; os, outer superior; sup, superior.
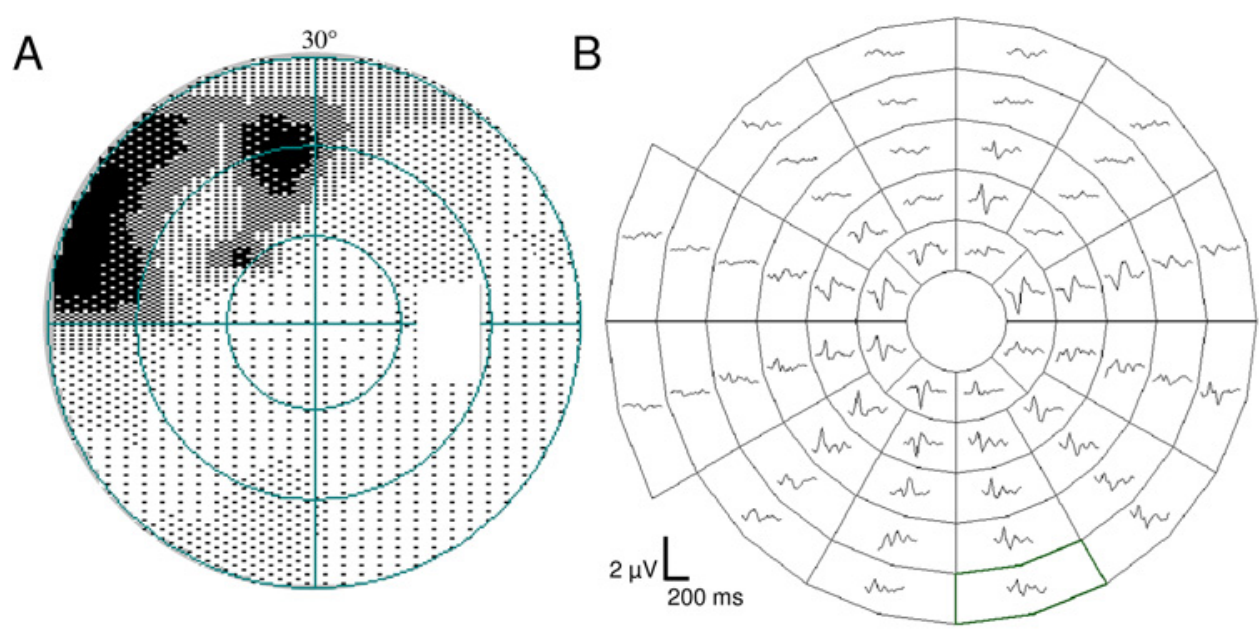

C

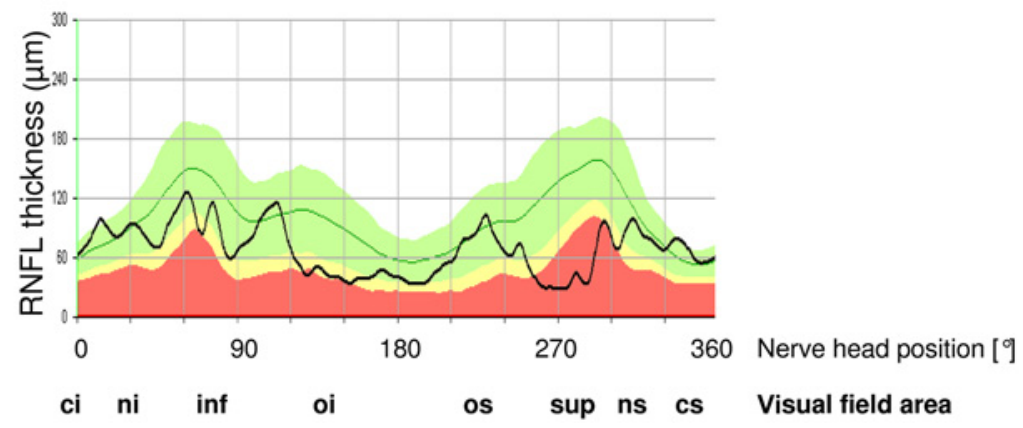


is $U_{M}=U_{A 0} \times 10^{-D / 10}+U_{R}$. In this paper, the residual voltage $U_{R}$ was calculated for each area as the mean of the amplitudes when local visual field losses were greater than $12 \mathrm{~dB}$.

To model a linear relationship between VEP amplitudes and corresponding RNFL thickness, least-square and Deming regressions were performed. The latter considers the variability of both measurements. ${ }^{21}$ The coefficients of variation for the SOCT were calculated using our own reliability measurements in 38 subjects and ranged between $3.2 \%$ (inf) and $4.9 \%$ (ci) for the measured sectors. The coefficient of variation of the mfVEP amplitudes was $10 \%$ for all visual field areas. This estimation is based on results from earlier studies ${ }^{22}$ and the observation ${ }^{4}$ that variability was similar for most stimulus locations except the more peripheral superior tests zones that are not considered in the present analysis.

\section{RESULTS}

The results from standard perimetry and mfVEP measurement in the same eye of a 31-year-old male patient with juvenile primary open angle glaucoma are shown as an example in figure 2. Visual field losses and reduced mfVEP amplitudes were found in the outer fields of the upper nasal quadrant (figure 2A,B). The thickness profile as measured with SOCT (figure $2 \mathrm{C}$ ) is given as a function of the position relative to the optic nerve head. Below the abscissa, the locations of the fibre bundle groups are shown. Considerable thinning of the RNFL thickness below the normal range can be seen in the peripapillar sector at about $270^{\circ}$, corresponding to the visual field defects in the superior field area as shown in figure $2 \mathrm{~A}$.

Figure 3 presents statistical VEP comparisons between controls and glaucoma patients in eight visual field areas introduced in figure 1 . The mean amplitudes in these nerve fibre

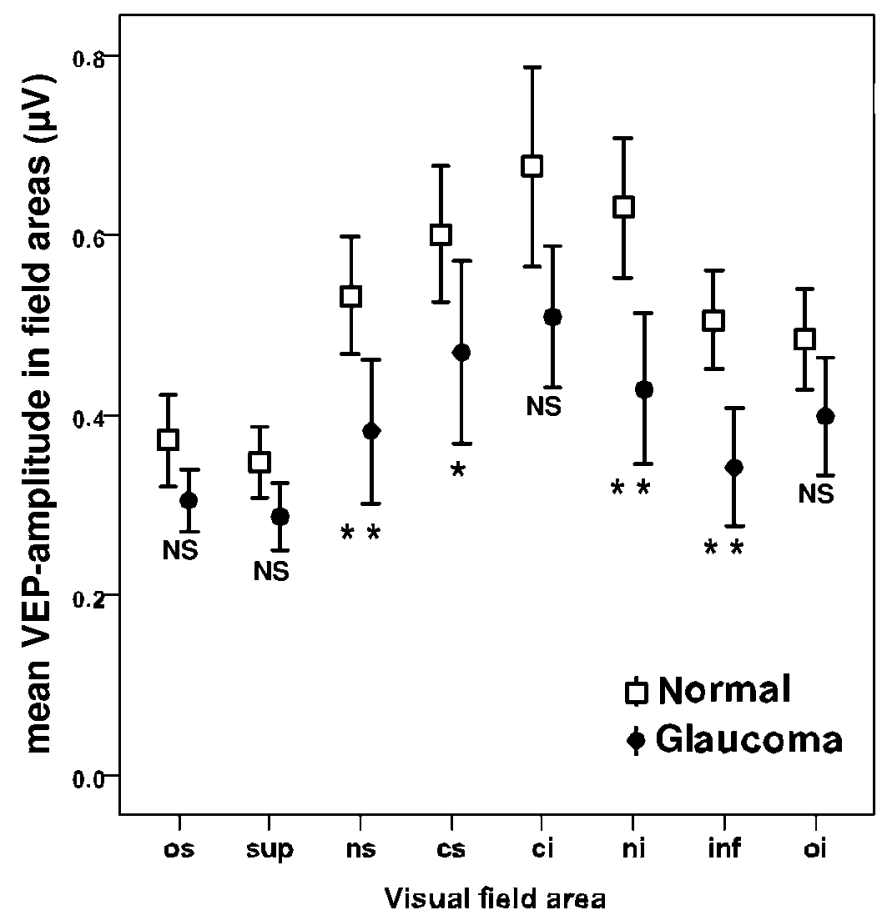

Figure 3 Mean amplitudes in visual field areas (mean $\pm 95 \% \mathrm{Cls}$ ) for the normal and glaucoma patient groups. The results of non-parametric comparisons with the Mann-Whitney $U$ test are included. Asterisks indicate significant differences between controls and patients ${ }^{*} p<0.05$, ${ }^{* *} \mathrm{p}<0.01$, NS, not significant; corrected for multiple comparison). related areas were between $0.35 \mu \mathrm{V}$ (superior area) and $0.68 \mu \mathrm{V}$ (central inferior area) for normal subjects and between $0.28 \mu \mathrm{V}$ and $0.51 \mu \mathrm{V}$, respectively, for glaucoma patients. The differences between both groups were significant in mid-peripheral areas but not for outermost areas and the central inferior area.

Figure 4 shows VEP amplitudes as a function of visual field defects (left column, A) and RNFL thickness (right column, B) for five visual field areas. The superior visual field area is not shown in figure 4 because there was no significant correlation between parameters. The plots in A and B show all individual data from glaucoma patients (filled symbols). The RNFL thickness plots (B) include the individual data from control subjects (open symbols), while for standard perimetry (A) data from normal subjects are given as mean VEP amplitude at $0 \mathrm{~dB}$.

The scatter-plots reveal that the VEP amplitudes decrease when visual field loss increases (A) and RNFL thickness decreases (B). The curve in (A) is a fit of the model, described in the Methods section, to the data. The model gives an adequate description of the data. The straight lines in (B) show results from least-square and Deming regressions, which gave similar results except for the central visual fields where the coefficient of variation from SOCT is relatively large. ${ }^{23}$ Significant Spearman rank coefficients were obtained between VEP amplitudes and visual field defects and between VEP amplitudes and RNFL thickness for the presented areas. To avoid an inhomogeneous correlation in the comparison, Spearman correlations were only performed on the patients' data.

\section{DISCUSSION \\ Measurement of mfVEPs}

The aims of this investigation were to establish an automated technique to record mfVEPs as an objective perimetric measure and to correlate the mfVEP data with standard perimetry and with RNFL thickness. Therefore, mfVEP measurements from nerve fibre bundle related areas were analysed in healthy subjects and glaucoma patients. The mean VEP amplitudes are decreased in glaucoma patients, particularly in those retinal areas that generally show larger vulnerability to glaucomatous damage: the Bjerrum area and the parafoveal region (figure 3 ). These results confirm earlier suggestions that analysing mfVEPs in Bjerrumlike sectors might be useful to increase performance and correlation with standard perimetry. ${ }^{24}$ Both groups in this study demonstrate the known amplitude asymmetry between upper and lower hemifields. ${ }^{25}$

\section{Correlation between VEP and other glaucoma tests}

The Spearman analyses reveal a high correlation between local VEP amplitudes and corresponding field losses (figure 4, left). The patients' VEP amplitudes decrease rapidly with increasing visual field defects (expressed in $\mathrm{dB}$ ), indicating that a small field loss results in a large VEP amplitude decrease. This is consistent with the observation of Hood et $a l^{26}$ that VEP amplitude is linearly related to perimetric visual field sensitivity. The data further indicate that the VEP signal can be considered as the sum of two components: the evoked potential and noise. The images suggest that elicited VEP potentials $U_{\mathrm{A}}$ are smaller than noiselevel $U_{R}$ when perimetric defects were larger than approximately $3 \mathrm{~dB}$. The same model has been used previously for the description of the linear relationship between perimetric sensitivity and RNFL thickness. ${ }^{17}{ }^{19}$ Consequently, the relationship between VEP responses and RNFL thickness can also be expected to be linear, which is confirmed by our data (figure 4, right column). 
Figure 4 Visual evoked potential (VEP) amplitudes as a function of visual field defects $(A)$ and retinal nerve fibre layer (RNFL) thickness (B) for five visual field areas. Left column: The VEP amplitudes (linear scale) decrease with increasing visual field loss ( $\mathrm{dB}$ scale) in glaucoma patients. A theoretical function (see the Statistical methods section) is included in each scatter-plot starting at the 'normal' mean amplitude and assuming a residual base-level $U_{R}$ if defects are worse than $12 \mathrm{~dB}$. Right column: VEP amplitudes plotted as a function of RNFL thickness (both in linear units). To model a linear relationship, the images include standard linear regression line (solid) and Deming regression (dotted line). Filled dots indicate glaucoma patients and open square symbols indicate normal controls; error bars in the left plots indicate the standard deviations in the control group. (Spearman correlation coefficients ${ }^{*} p<0.05$, ${ }^{* *} \mathrm{p}<0.01$, adjusted for multiple comparison).
A
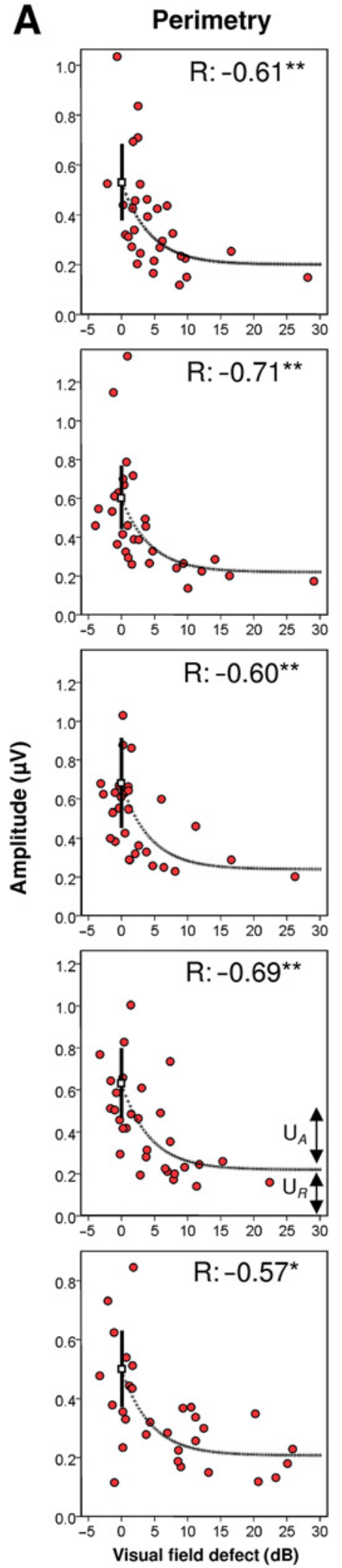

B

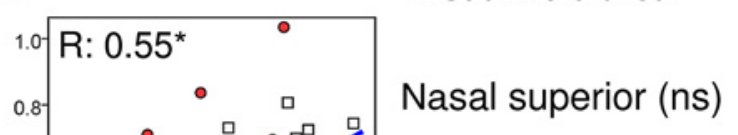

Visual field area:

Nasal superior (ns)
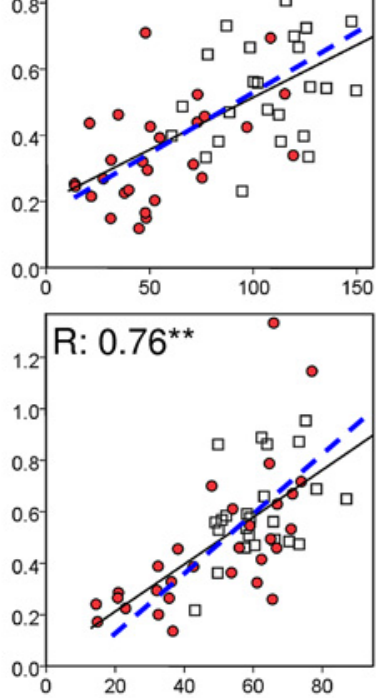

Central superior (cs)

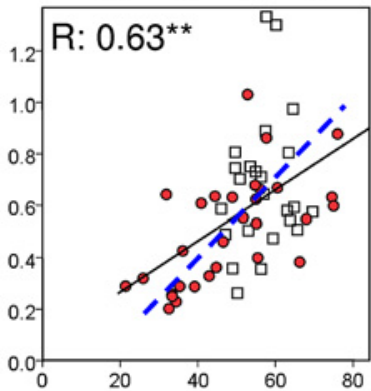

Central inferior (ci)

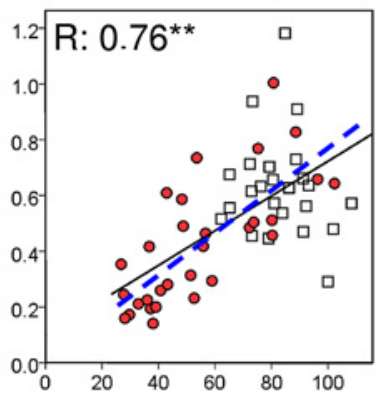

Nasal inferior (ni)

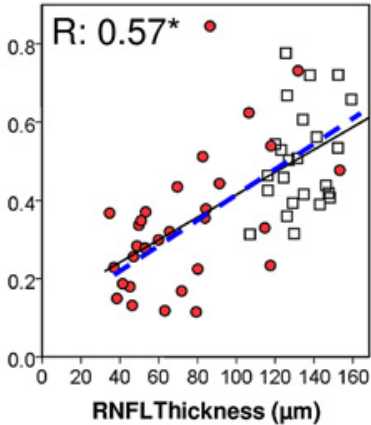

Inferior (inf)
Baseline data and the relationship between mfVEP data and other techniques might be influenced by the acquisition and analysis techniques. The present electrode configuration relative to the cortical electric dipole ensured large VEP responses. The recording with the highest SNR was automatically chosen to represent the response to the relevant visual field. Future studies with more sophisticated filter methods ${ }^{27} 28$ might be able to optimise the ratio between the evoked signal and the base level. A drawback of the mfVEP technique as an objective perimetry method is the relatively long recording time necessary for reliable data and the possible influence of movements or muscle tension. Approximately $15 \%-20 \%$ of the mfVEP measurements do not yield interpretable results when a minimal SNR of 1.4 is applied for data inclusion. This drop-out rate has to be compared 
with the number of non-reliable measurements in conventional perimetry due to false positive or negative responses. In addition, the short- and long-term reproducibility ${ }^{29-31}$ of the methods must be compared.

\section{CONCLUSIONS}

Results in topographically related areas show a linear relationship between local mfVEP responses and corresponding RNFL thickness as well as visual field defects. Even relatively small perimetric losses can lead to considerable reduction in the evoked VEP signal. Thus, the present automated method can reveal many damaged locations in the visual field of glaucoma eyes.

Funding This work was supported by the German Research Council (SFB539).

Competing interests None.

Ethics approval The study was approved by the Local Ethics Committee.

Contributors All authors: (1) conceived and designed the study or analysed and interpreted the data; (2) wrote the manuscript or provided critical revisions that are important for the intellectual content; and (3) approved the final version of the manuscript.

Provenance and peer review Not commissioned; externally peer reviewed.

\section{REFERENCES}

1. Odom JV, Bach $M$, Brigell $M$, et al. ISCEV standard for clinical visual evoked potentials (2009 update). Doc Ophthalmol 2010;120:111-19.

2. Baseler HA, Sutter EE, Klein SA, et al. The topography of visual evoked response properties across the visual field. Electroencephalogr Clin Neurophysiol 1994;90:65-81.

3. Hood DC, Zhang $X$, Hong JE, et al. Quantifying the benefits of additional channels of multifocal VEP recording. Doc Ophthalmol 2002;104:303-20.

4. Klistorner A, Graham SL. Objective perimetry in glaucoma. Ophthalmology 2000:107:2283-99.

5. Hood DC, Zhang X, Greenstein VC, et al. An interocular comparison of the multifocal VEP: a possible technique for detecting local damage to the optic nerve. Invest Ophthalmol Vis Sci 2000:41:1580-7.

6. Hood DC, Odel JG, Zhang $X$. Tracking the recovery of local optic nerve function after optic neuritis: a multifocal VEP study. Invest Ophthalmol Vis Sci 2000;41:4032-8.

7. Graham SL, Klistorner Al, Goldberg I. Clinical application of objective perimetry using multifocal visual evoked potentials in glaucoma practice. Arch Ophthalmol 2005;123:729-39.

8. Watanabe K, Shinoda K, Kimura I, et al. Discordance between subjective perimetric visual fields and objective multifocal visual evoked potential-determined visual fields in patients with hemianopsia. Am J Ophthalmol 2007:143:295-304.

9. Kaltwasser C, Horn FK, Kremers J, et al. Objective visual field determination in forensic ophthalmology with an optimized 4-channel multifocal VEP-perimetry system: a case report of a patient with retinitis pigmentosa. Doc Ophthalmol 2011;123:121-5.
10. Punjabi OS, Stamper RL, Bostrom AG, et al. Topographic comparison of the visual function on multifocal visual evoked potentials with optic nerve structure on heidelberg retinal tomography. Ophthalmology 2008;115:440-6.

11. Klistorner A, Arvind $H$, Nguyen $T$, et al. Multifocal VEP and OCT in optic neuritis: a topographical study of the structure-function relationship. Doc Ophthalmol 2009:118:129-37.

12. Laron M, Cheng $\mathrm{H}$, Zhang B, et al. Comparison of multifocal visual evoked potential standard automated perimetry and optical coherence tomography in assessing visual pathway in multiple sclerosis patients. Mult Scler 2010:16:412-26.

13. Hood DC, Greenstein VC. Multifocal VEP and ganglion cell damage: applications and limitations for the study of glaucoma. Prog Retin Eye Res 2003:22:201-51.

14. Horn FK, Wakili N, Junemann AM, et al. Testing for glaucoma with frequencydoubling perimetry in normals, ocular hypertensives, and glaucoma patients. Graefes Arch Clin Exp Ophthalmol 2002;240:658-65.

15. Jonas JB, Gusek GC, Naumann GO. Optic disc morphometry in chronic primary open-angle glaucoma. I. Morphometric intrapapillary characteristics. Graefes Arch Clin Exp Ophthalmol 1988:226:522-30.

16. Bendschneider D, Tornow RP, Horn FK, et al. Retinal nerve fiber layer thickness in normals measured by spectral domain OCT. J Glaucoma 2010;19:475-82.

17. Horn FK, Mardin CY, Laemmer R, et al. Correlation between local glaucomatous visual field defects and loss of nerve fiber layer thickness measured with polarimetry and spectral domain OCT. Invest Ophthalmol Vis Sci 2009;50:1971-7.

18. Horn FK, Mardin CY, Baleanu D, et al. Analysis of peripapillary nerve fiber layer thickness (assessed by SOCT) with automated classification and correction for individual optic disk locations. ARVO Meeting Abstracts 2010;51:260.

19. Hood DC, Kardon RH. A framework for comparing structural and functional measures of glaucomatous damage. Prog Retin Eye Res 2007;26:688-710.

20. Hood DC, Anderson SC, Wall M, et al. Structure versus function in glaucoma: an application of a linear model. Invest Ophthalmol Vis Sci 2007;48:3662-8.

21. Linnet K. Evaluation of regression procedures for methods comparison studies. Clin Chem 1993:39:424-32.

22. Klistorner Al, Graham SL. Multifocal pattern VEP perimetry: analysis of sectoral waveforms. Doc Ophthalmol 1999;98:183-96.

23. Mansoori T, Viswanath K, Balakrishna N. Reproducibility of peripapillary retinal nerve fibre layer thickness measurements with spectral domain optical coherence tomography in normal and glaucomatous eyes. Br J Ophthalmol 2011:95:685-8.

24. Graham SL, Klistorner A, Grigg JR, et al. Objective perimetry in glaucoma: recent advances with multifocal stimuli. Surv Ophthalmol 1999;43(Suppl 1):S199-209.

25. Fortune B, Hood DC. Conventional pattern-reversal VEPs are not equivalent to summed multifocal VEPs. Invest Ophthalmol Vis Sci 2003;44:1364-75.

26. Hood DC, Greenstein VC, Odel JG, et al. Visual field defects and multifocal visual evoked potentials: evidence of a linear relationship. Arch Ophthalmol 2002;120:1672-81.

27. Lindenberg T, Peters A, Horn FK, et al. Diagnostic value of multifocal VEP using cross-validation and noise reduction in glaucoma research. Graefes Arch Clin Exp Ophthalmol 2004;242:361-7.

28. Miguel-Jimenez JM, Ortega S, Boquete L, et al. Multifocal ERG wavelet packet decomposition applied to glaucoma diagnosis. Biomed Eng Online 2011;10:37.

29. Chen CS, Hood DC, Zhang $X$, et al. Repeat reliability of the multifocal visual evoked potential in normal and glaucomatous eyes. J Glaucoma 2003:12:399-408.

30. Fortune B, Demirel S, Zhang X, et al. Repeatability of normal multifocal VEP: implications for detecting progression. J Glaucoma 2006;15:131-41.

31. Punjabi OS, Stamper RL, Bostrom AG, et al. Repeatability of the multifocal visual evoked potentials in a clinical glaucoma setting. Can J Ophthalmol 2008;43:435-40. 


\section{B. Objective perimetry using a four-channel multifocal VEP system: correlation with conventional perimetry and thickness of the retinal nerve fibre layer}

Folkert K Horn, Christoph Kaltwasser, Anselm G Jünemann, Jan Kremers and Ralf $P$ Tornow

Br J Ophthalmol 2012 96: 554-559 originally published online November 24, 2011

doi: 10.1136/bjophthalmol-2011-300844

Updated information and services can be found at:

http://bjo.bmj.com/content/96/4/554

\section{These include:}

References This article cites 31 articles, 9 of which you can access for free at: http://bjo.bmj.com/content/96/4/554\#BIBL

Email alerting Receive free email alerts when new articles cite this article. Sign up in the service box at the top right corner of the online article.

Topic Articles on similar topics can be found in the following collections Collections

Angle (945)

Glaucoma (930)

Intraocular pressure (942)

\section{Notes}

To request permissions go to:

http://group.bmj.com/group/rights-licensing/permissions

To order reprints go to:

http://journals.bmj.com/cgi/reprintform

To subscribe to BMJ go to:

http://group.bmj.com/subscribe/ 